\title{
The Neurological Foundation of Geometry
}

\author{
Kazuhiko Kotani \\ Department of Psychiatry, Sakamoto Hospital, Higashiosaka, Japan \\ Email: kotanikazuhiko@gmail.com
}

How to cite this paper: Kotani, K. (2020). The Neurological Foundation of Geometry. Open Journal of Philosophy, 10, 143-154. https://doi.org/10.4236/ojpp.2020.101011

Received: January 3, 2020

Accepted: February 8, 2020

Published: February 11, 2020

Copyright (c) 2020 by author(s) and Scientific Research Publishing Inc. This work is licensed under the Creative Commons Attribution International License (CC BY 4.0).

http://creativecommons.org/licenses/by/4.0/

\begin{abstract}
Geometry is based on vision. Hence, the visual information processing of the nervous system regulates the structure of geometry. In this paper, we shall construct geometry following the process of visual information processing in the nervous system. Firstly, photons are captured by photoreceptor cells in the retina. At this stage, the retinal bitmap image is constructed using photoreceptor cells as pixels. The retinal bitmap is the foundation of quantitative properties of images throughout the visual processing. Secondly, the edge of the object is extracted in the primary visual cortex. When a three-dimensional object is projected in two dimensions, the edge of the object is ideally a line without width. While Euclid defined the line as the length without width. Surprisingly, a type of cells in the primary visual cortex react the Euclidean line. At this stage, Euclidean geometry without curves is constructed. Thirdly, curves are recognized in the visual area V4. At this stage, Euclidean geometry with curves is constructed. The next problem is the compatibility of these stages. The problem of the compatibility between the first and second stages is that there are irrational lengths in Euclidean geometry. Ancient Greeks used the double contradiction to solve the compatibility problem. An irrational number is defined as a number that divides rational numbers into larger and smaller rational numbers. The double contradiction is a method of defining non-rational numbers using rational numbers. Also, double contradiction is used to solve the compatibility problem between the second and third stages. Even though the length of the curve is not defined in Elements, the length of a curve can be defined by the length of straight lines. Similarly, properties of curves are defined by straight lines. In differentiation, the slope of the curve is defined by the slope of the line. In integration, the area under the curve is defined by the total area of thin rectangles. Finally, as a logical basis for calculus, the double contradiction should be rethought.
\end{abstract}

\section{Keywords}

Geometry, Neuroscience, Vision, Calculus, Double Contradiction, Elements 


\section{Introduction}

Ancient Greeks thought that the natural number one is the basic concept. Especially, Plato emphasized the importance of the natural number one. Furthermore, Plato described three basic properties of the natural number one: equality, indivisibility, invariability (Plato, 2007). Surely, Plato regarded the natural number one as the base of cognition.

On the other hand, neuroscience has advanced recently, and the neural circuit, which is the source of mathematics, is gradually being elucidated. Stanislas Dehaene, a neuroscientist and a mathematician, states as follows (Dehaene, 2011):

The foundations of any mathematical construction are grounded on fundamental intuitions such as notions of set, number, space, time, or logic. These are almost never questioned, so deeply do they belong to the irreducible representations concocted by our brain. Mathematics can be characterized as the progressive formalization of these intuitions. Its purpose is to make them more coherent, mutually compatible, and better adapted to our experience of the external world.

So, because human intuition is made by evolution, it is difficult to make consistency between two other intuitions. Our intuitions have been shaped in the history of evolution for survival, not logically structured. Distortion tends to occur at the joint of two intuitions. Dehaene says that we should strive to minimize distortion. Now, neuroscience has advanced. Then, we can consider the neurological basis of each intuition. We should reconsider the foundation of mathematics based on neuroscience. Since visual information processing is fairly well understood (Banich \& Compton, 2018), we focus on geometry. We shall build geometry based on the thought of Dehaene.

In this paper, we choose three intuitions: the pixel, the Euclidean line and the curve. These intuitions correspond to three stages of visual processing respectively. In accordance with the order of visual information processing, the correspondence between intuition, visual information processing stage and anatomical structure is described. Firstly, the pixel corresponds to the capture of photons in the retina. Secondly, the Euclidean line corresponds to the extraction of the edge in the primary visual cortex. Thirdly, the curve corresponds to the extraction of the contour of the object in the extrastriate visual cortex.

We shall consider each intuition and the relationship among intuitions. However, since the base of all considerations is the natural number one, we shall start with consideration of the natural number one (Kotani, 2017).

\section{The Natural Number One}

When we assume that the natural number one is the base of the cognition, the next question has arisen. What is the substantial entity of the natural one? This question is difficult to answer. However, the most familiar and the most prom- 
ising candidate of the answer is a human being oneself. Firstly, a human being is indivisible. If a human being is divided, the human being will die or survive with serious injuries. One person cannot be divided into two persons. Because of the indivisibility, a human being can be regarded as the prototype of the natural number one.

Human beings are indivisible. Also, higher animals are indivisible. However, there are many plants that can be divided. Furthermore, multicellular organisms can be divided into a single cell in principle. So, many human cells can be cultured. Even though a human being dies, modern technology can save organs and cells of the human being. Therefore, the minimum unit of life is the cell (Alberts, Johnson, Lewis, Raff, Roberts, \& Walter, 2002a), and the cell is indivisible. Necessarily, we can count the number of cells. At least a cell has the indivisible property of the natural number one.

As a result of the above considerations, strictly speaking, the cell is the unit of life. However, when we consider humans, we always regard individuals as units. Similarly, for mammals such as livestock and pets, we tend to regard individuals as units. For these reasons, it is easier for us to consider a life itself as a unit than to consider a cell as a unit. Although it may be somewhat less rigorous, we will continue to consider a life as a unit.

Plato said that the natural number one is equal to each other, invariable and indivisible. This description can be regarded as the definition of the natural number one. So far, it is certain that life is indivisible. Life satisfies this definition. However, what does life mean to be unchanged and equal to each other? Differences in lives are due to differences of genetic information but all lives have the common genetic information, which are assumed to have been conserved from the last universal common ancestor of all life (Alberts, Johnson, Lewis, Raff, Roberts, \& Walter, 2002b). Especially, proteins required for transcription and translation are highly conserved. There are many common features of all life, but the remarkable feature is trying to survive and to proliferate. Another important feature is dying. If the genetic differences are removed, we can say that all lives are invariable and equal to each other. In this way, life has properties of the natural number one.

In addition, we shall consider the basics of our perception. Necessarily, a cell is the basis of recognition. Furthermore, neurons have the ability to transmit signals to other neurons, and the main signal of the neuron is the action potential, which has the properties of the natural number one (Kotani, 2017). The action potential is indivisible, invariable and equal to each other. So, the action potential is almost identical, regardless of the type of the neuron and the specie of the animal (Nichollis, Martin, Wallace, \& Fuchs, 2002a). Therefore, the basis of our recognition is the natural number one.

\section{From Eyes to the Primary Visual Cortex}

Natural numbers have long been recognized as the most reliable intuition, and 
ancient Greeks tried to construct mathematics based on natural numbers. This fact is inferred from Plato's writing and Euclid's Elements (Euclid, 1956b; Plato, 2007). However, their trial was unsuccessful, and Euclidean geometry was built on axioms. Also, geometry was built on axioms in modern mathematics (Hilbert, 1950). However, the base of recognition is the natural number in our nervous system. Therefore, if we consider how our visual nervous system creates the visual image, we can approach the constructive geometry.

In this paper, we shall examine the foundation of geometry tracing information processing in the visual nervous system. Geometry is based on the world seen by human beings. On the other hand, it is assumed that the ancient Greeks thought deeply about human vision and built geometry based on it. Surprisingly, the definition of the line in Euclid's Elements corresponds to the reactivity of a certain type of cells in the primary visual cortex (Euclid, 1956a; Nichollis, Martin, Wallace, \& Fuchs, 2002b). In this way, the structure of Euclid's Elements is closely related to the visual information processing. However, curves are not quantitatively handled in Euclid's Elements. Therefore, we shall mainly consider Euclidean geometry without curves in the first half of this paper. Curves will be considered later in the second half of this paper. Then, we shall survey the visual processing from eyes to the primary visual cortex.

In the beginning, photoreceptor cells capture photons (Tessier-Lavigne, 2000). At this stage, the retinal bitmap image, which consists of pixels as photoreceptor cells, is obtained, and we can construct the bitmap geometry. Furthermore, the retinal bitmap image is the base of quantitative properties of visual images.

Subsequently, information is processed in the retina to enhance the contrast between light and dark. The converted information continues to enter the primary visual cortex in the occipital lobe of the cerebrum through the visual conduction pathway.

\section{The Creation of the Breadthless Line in Our Brain}

Next, the edge of the object is extracted in the primary visual cortex, and the recognition of the shape of the 3D object begins (Nichollis, Martin, Wallace, \& Fuchs, 2002b). Simultaneously, the image of the object is converted from digital to analog because analog images with free scaling are advantageous for recognizing moving objects. For the conversion from digital to analog, pixels are hidden and the number of pixels is converted to the length of the line in the primary visual cortex.

Why is the shape of an object so important? Moving objects can be prey or predators. Suppose an animal sees an object. If the object is a predator, the animal must run away. Conversely, if the object is prey and the animal is hungry, the animal tries to catch it. As just mentioned, the animal needs to identify the object in order to survive. Once the object is identified, the animal can determine how to deal with the object. Thus, object identification is important $(\mathrm{Pa}$ supathy, 2015). 
As the first step in object identification, the edges of the object are extracted in the primary visual cortex. Figure 1 shows the cube and its shadow. As shown in Figure 1, the edge of the object corresponds to the borderline between light and dark.

In the primary visual cortex, there are a type of cells, which react to the line segment between light and dark at a specific position and at a specific angle (Hubel \& Wiesel, 1959; Hubel \& Wiesel, 1968). While, the primary visual cortex cell arrangement corresponds to the retinal cell arrangement, and the relative positional relationship is preserved. This stage is important for Euclidean geometry.

Next, if the borderline between light and dark is idealized, its width should be zero. So, it is identical to the Euclidean line. While, the definition of the line is the most elemental definition in Elements (Euclid, 1956a). Subsequently, a point is defined as the ends of a line in Elements. Definitions 1 to 3 in Elements are as follows.

Definition 1. A point is that which has no part.

Definition 2. A line is breadthless length.

Definition 3. The ends of a line are points.

Now, we notice that the straight borderline between light and dark corresponds to the line defined by Euclid.

\section{The Linear Euclidean Geometry}

Once a line is defined, Euclidean geometry without curves can be constructed. Then, we shall call Euclidean geometry without curves the linear Euclidean geometry. While, the ancient Greeks thought that the basic element of the world was the natural number one, so they thought that lines were composed of natural numbers. Hence, it is assumed that they had been constructed the bitmap geometry, in which lines are composed of pixels. However, because the width of the pixelated line is not zero, pixelated line cannot be the Euclidean line. Figure 2(1) shows the Euclidean line and Figure 2(2) shows the pixelated line.

Thus, the compatibility between the linear Euclidean geometry and the bitmap

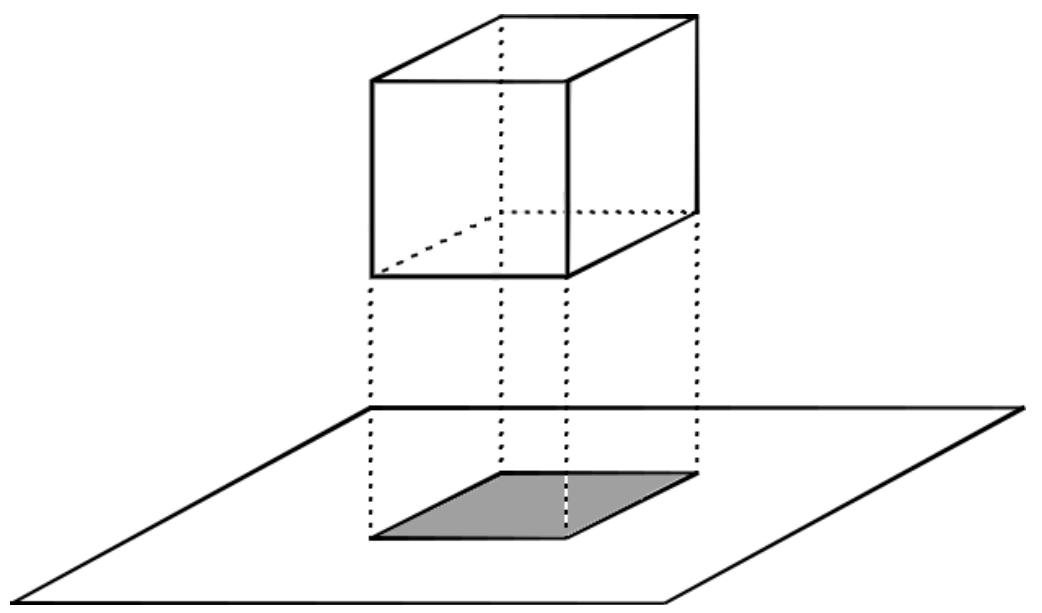

Figure 1. The square shadow of the cube. 


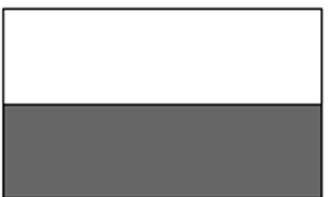

(1)

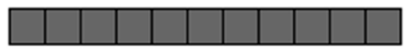

(2)

Figure 2. The Euclidean line and the pixelated line.

geometry is incomplete. In fact, Pythagoras found a Euclidean line with a length that had no ratio to the reference. The discovery of the irrational number is a big shock for ancient Greeks. However, if we reduce the size of pixels, we can approximate the Euclidean line by the pixelated line as precise as we want. So, Eudoxus developed the double contradiction, which corresponds to definition 5 in book 5 of Elements (Heath, 2013). It is identical to Dedekind's cut, which defines an irrational number by rational numbers.

\section{The Problem of the Curve}

The next problem is the length of the curve. Euclid did not define the length of the curve while he defined the line as the breadthless length. Because the shortest distance between two points is the length of the straight line between them, the length generally means the length of the line segment. That is, the concept of length is deeply tied to the straight line. Thus, the concept of the length of the curve itself is problematic. It is controversial whether to accept lines with length undefined. So, it is difficult to keep the coherence between the straight line, which is the length itself, and the curve with length undefined.

The shape of the object is recognized in the extrastiate cortex (Wurtz \& Kandel, 2000). Because many objects have the curvature contour, the recognition of it is important for the identification of the object. Even though the process of the shape recognition is complicated and many areas are involved in the shape recognition, the area V4 is presumed to play a central role in the recognition of the curve (El-Shamayleh \& Pasupathy, 2016; Pasupathy, 2015; Yue, Pourladian, Tootell, \& Ungerleider, 2014).

The relationship between the retinal bitmap and the curve is indirect because the curve is recognized in higher areas. On the other hand, the primary visual cortex is received input from the retina. So, the straight borderline between light and dark can maintain the direct correspondence to the retinal bitmap. As shown in Figure 3, the relationship between the retinal bitmap and the straight borderline between light and dark is obvious. Figure 3(1) shows the straight borderline between light and dark, and Figure 3(2) shows the retinal bitmap. The correspondence between the length of the straight line and the number of pixels is obvious. Therefore, it is natural to try to approximate the length of the curve by line segments.

In fact, Archimedes developed the method of the approximation of pi. The circumference is longer than the perimeter of an inscribed regular polygon and 


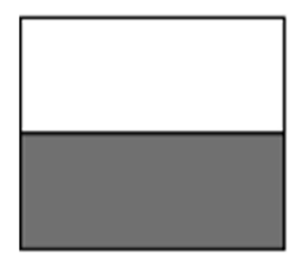

(1)

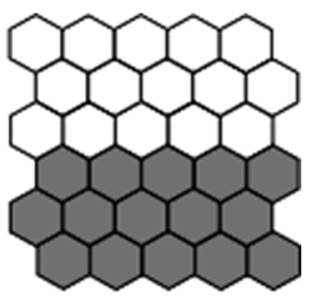

(2)

Figure 3. The straight borderline between light and dark, and the retinal bitmap.

is shorter than the perimeter of a circumscribed regular polygon. When we calculate the length of the perimeter of the inscribed polygon and the length of the perimeter of the circumscribed polygon, we can sandwich the circumference by them. Archimedes duplicated the number of sides of the regular polygon from hexagon to 96-sided regular polygon (Archimedes, 2002). He iterated calculation of the perimeter of polygons. Finally, he obtained the approximated value of pi. Using Archimedes' method, we can approximate pi as precise as we want. Therefore, pi is a Dedekind cut that divides a rational number into two sets.

\section{The Integral Calculus Based on the Double Contradiction}

Naturally, quantitative properties of the curve, including the length of the curve, could only be defined using straight lines. Hence, the double contradiction is necessary to provide the logical foundation of handling curves quantitatively. To make the double contradiction more familiar to us, we use an integration of a simple monotonically increasing function.

For example, Figure 4 shows the approximation of the area $S$. We shall consider the area under the graph of $y=x^{2}$ in the interval from 0 to 1 . It is named $\mathrm{S}$. Equation (1) represents the integral of $y=x^{2}$ from 0 to 1.

$$
S=\int_{0}^{1} x^{2} \mathrm{~d} x
$$

The area $\mathrm{S}$ is approximated by $\mathrm{n}$ thin rectangles. The width of each rectangle is $1 / n$. The left side of Figure 4 shows the right sum, in which rectangles touch the curve with their top-right corners. Equation (2) represents the right sum:

$$
\frac{1}{n} \sum_{m=1}^{n}\left(\frac{m}{n}\right)^{2}=\frac{1}{n}\left[\left(\frac{1}{n}\right)^{2}+\left(\frac{2}{n}\right)^{2}+\cdots+\left(\frac{n}{n}\right)^{2}\right]=\frac{1}{3}+\frac{1}{2 n}+\frac{1}{6 n^{2}} .
$$

The right side of Figure 4 shows the left sum, in which rectangles touch the curve with their top-left corners. Equation (3) represents the left sum:

$$
\frac{1}{n} \sum_{m=0}^{n-1}\left(\frac{m}{n}\right)^{2}=\frac{1}{n}\left[\left(\frac{0}{n}\right)^{2}+\left(\frac{1}{n}\right)^{2}+\cdots+\left(\frac{n-1}{n}\right)^{2}\right]=\frac{1}{3}-\frac{1}{2 n}+\frac{1}{6 n^{2}}
$$

Because $y=x^{2}$ is monotonically increasing, the left sum is smaller than $S$, and the right sum is larger than $S$. Then, inequality (4) is established:

$$
\frac{1}{3}-\frac{1}{2 n}+\frac{1}{6 n^{2}}<S<\frac{1}{3}+\frac{1}{2 n}+\frac{1}{6 n^{2}} \text {. }
$$



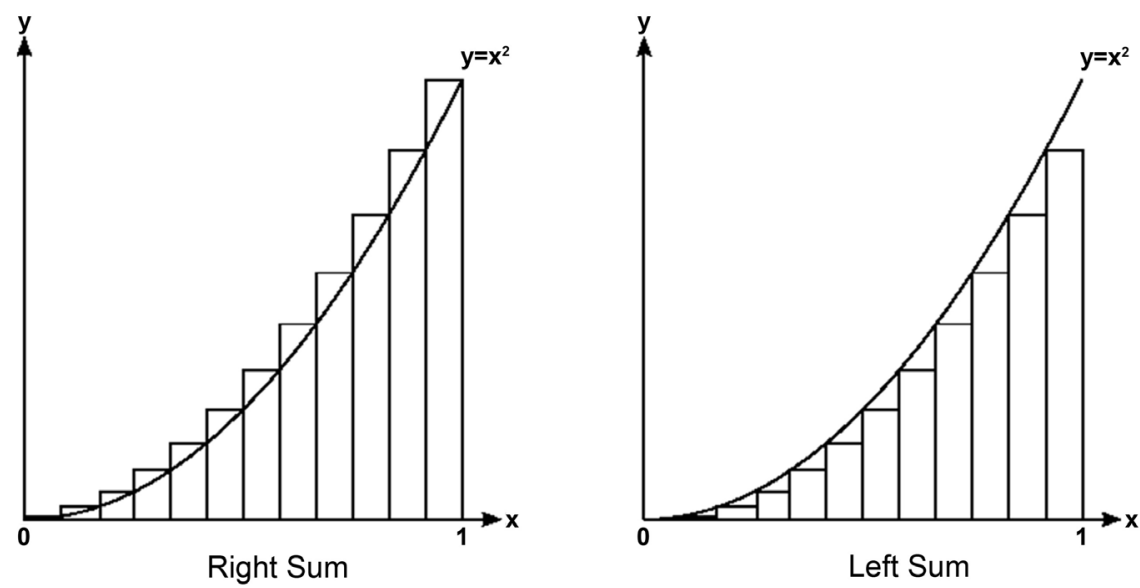

Figure 4. The right sum and the left sum.

Because $\mathrm{n}$ is an arbitrary natural number, if $S$ is larger or smaller than $1 / 3$, the contradiction occurs. Then, Equation (5) is established:

$$
S=\frac{1}{3} \text {. }
$$

A simple example of the double contradiction is shown above. The double contradiction used by the ancient Greeks is logically rigorous. It provides the rigorous basis of the calculus. Its significance will be discussed later.

\section{The Differential Calculus Based on the Double Contradiction}

The next problem is the differential calculus. Figure 5 shows the double contradiction for the differential calculus of a convex curve $y=f(x)$ (Kotani, 2016). $L$ (right) intersects the curve at the right side of point $A$ and point $A . L($ left) intersects the curve at the left side of point $A$ and point $A . L(A)$ is the tangent at point $A$. Slopes of each line are represented $S$ (left), $S(A), \mathrm{S}$ (right), respectively. Slopes of lines have the following relationship:

$$
S(\text { left })<S(A)<S \text { (right). }
$$

when the $x$ coordinate of the left intersection point is $x-g$ and the $x$ coordinate of the right intersection point is $x+h, S($ left $)$ and $S$ (right) are represented by Equation (7):

$$
\begin{aligned}
& S(\text { left })=\frac{f(x)-f(x-g)}{g}, \\
& S(\text { right })=\frac{f(x+h)-f(x)}{h} .
\end{aligned}
$$

Substitute the right sides of Equation (7) into inequality (6):

$$
\frac{f(x)-f(x-g)}{g}<S(A)<\frac{f(x+h)-f(x)}{h} .
$$

Because $g$ and $h$ in the inequality (8) are arbitrary positive real numbers, the slope 


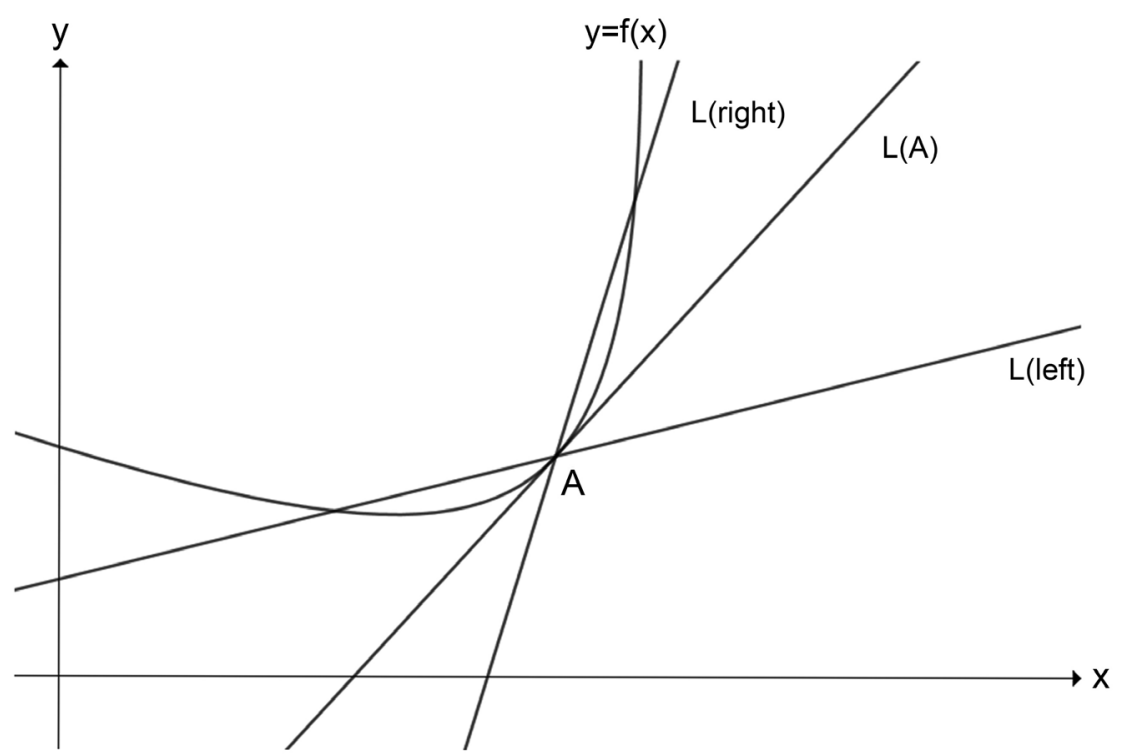

Figure 5. The double contradiction for the differential calculus of a convex curve.

of the tangent at point $A$ is determined as $S(A)$ using the double contradiction.

Next, to make the double contradiction more familiar to us, we shall determine the slope at point $A$ of a simple function $y=x^{2}$ using the double contradiction. When the coordinates of the point $A$ is $\left(x, x^{2}\right), S($ left $)$ and $S$ (right) are as follows:

$$
\begin{aligned}
& S(\text { left })=\frac{x^{2}-(x-g)^{2}}{g}=2 x-g, \\
& S(\text { right })=\frac{(x+h)^{2}-x^{2}}{h}=2 x+h .
\end{aligned}
$$

Because $y=x^{2}$ is monotonically increasing, inequality (10) is established:

$$
2 x-g<S(A)<2 x+h .
$$

Because $g$ and $h$ in the inequality (10) are arbitrary positive real numbers, the slope of the tangent at the point $A$ is determined as $S(A)$ using the double contradiction.

$$
S(A)=2 x .
$$

As shown in Equation (11), the double contradiction can be applied to differentiation.

\section{Discussion}

Firstly, when we are seeing, the retinal bitmap image is configured. At this stage, there is a bitmap image with photoreceptor cells as pixels. Thus, the image in the retina is digital. That is, the retinal image is the data, which consists of natural numbers. Furthermore, the positional relationship among photoreceptor cells is conserved throughout the visual nervous system. That is, the retinal bitmap image is the base of quantitative properties of visual images. Therefore, the quan- 
titative properties of visual images are based on digital information.

Secondly, the retinal digital image is converted to the analog image in the primary visual cortex. At this stage, a line without width corresponding to the edge of the object is formed. It is identical to the breadthless length, which is defined in Elements.

However, if we think more deeply, we notice that we cannot see the continuous line without width. Our brains just make us feel that we see it. However, the resolution of the eye is determined by the size of the photoreceptor cells. So, just because the brain is hiding the pixels, we feel to see the analog image. Instead, what we are seeing is really the pseudo-analog image. If so, Euclidean geometry is a pure creation of ancient Greeks. Perhaps the ancient Greeks had developed geometry based on their deep analysis of vision. Furthermore, it is assumed that ancient Greeks gave universality to Euclidean geometry through abstraction and idealization, which are features of their thought. Among their creations, the Euclidean line is a masterpiece. When a $3 \mathrm{D}$ object is projected in two dimensions, ideally the edge of the 3D object becomes the Euclidean line.

Thirdly, the curve is recognized in higher visual areas. Thus, it is presumed that a very large number of nerve cells are involved in the recognition of curves. While, there are approximately 120 million rods and 6 million cones in the human retina (Banich \& Compton, 2018). Rods and Cones are photoreceptor cells. With so many cells involved, neurons in lower visual areas may not be able to recognize curves just as we cannot recognize the roundness of the earth in our daily lives. Curves are also considered important for recognizing the overall characteristics of the $3 \mathrm{D}$ object. Necessarily, the quantification of the curve is difficult.

Next, we shall consider the double contradiction in detail. An example is shown as follows. We shall start by trying to approximate an irrational number by rational numbers. However, no matter how close rational numbers are, they cannot reach the irrational number. Approximation from the larger and smaller sides is the same. We usually give up here, but here we reverse the idea. That is, we define an irrational number rather than trying to approximate it. Consider an arbitrary rational number. It is larger or smaller than the irrational number. This definition is identical to Dedekind's cut. It is important to note that non-rational numbers are defined using only rational numbers. In this way, we can introduce irrational numbers, which have never been. In other words, the world of numbers is expanded by the double contradiction.

The following expansion of the world of numbers by the double contradiction is an approximation of Pi by Archimedes. Archimedes' method can be repeated as many times as we want, and we can approximate the pi to any accuracy. Then, we can define pi with the double contradiction. In this case, the length of the curve can be defined using the length of the straight line. As a result, the world of numbers is expanded by the double contradiction.

Surely, the double contradiction provides the rigorous basis for the quantifica- 
tion of curves. We should use the double contradiction whenever possible. However, there are technically difficult cases. Calculations tend to be complicated in integration. Thus, only a simple example of integration is introduced in this paper. Nevertheless, we can apply the double contradiction to differentiation. The function can be differentiated using the double contradiction for a convex interval or a concave interval of the graph. The double contradiction provides the rigorous and intuitive basis for differentiation.

\section{Conclusion}

We have considered the foundation of geometry according to the idea of Dehaene. The geometry constructed from each intuition, corresponds to each anatomical structure. In this paper, we examined three stages of the visual processing in the visual nervous system. At the first stage, the bitmap geometry corresponds to the retina. At the second stage, the linear Euclidean geometry corresponds to the primary visual cortex. At the third stage, the Euclidean geometry with curves corresponds to the extrastriate visual cortex. As Dehaene pointed out, there is a gap between a stage and the next stage. However, Ancient Greeks developed the double contradiction. Using the double contradiction, we can maintain the quantitative compatibility between a stage and the next stage.

\section{Conflicts of Interest}

The authors declare no conflicts of interest regarding the publication of this paper.

\section{References}

Archimedes (2002). Measurement of a Circle. In The Works of Archimedes (pp. 91-97, Heath, T.L., Trans.). Mineola, NY: Dover Publications, Inc. https://doi.org/10.1017/CBO9780511695124.012

Banich, M. T., \& Compton, R. J. (2018). Sensation and Perception. In Cognitive Neuroscience (4th ed. pp. 136-164). Cambridge: Cambridge University Press. https://doi.org/10.1017/9781316664018

Alberts, B., Johnson, A., Lewis, J., Raff, M., Roberts, K., \& Walter, P. (2002a). The Universal Features of Cells on Earth. In Molecular Biology of the Cell (4th ed., pp. 3-13). New York: Garland Science.

Alberts, B., Johnson, A., Lewis, J., Raff, M., Roberts, K., \& Walter, P. (2002b). The Diversity of Genomes and the Tree of Life. In Molecular Biology of the Cell (4th ed., pp. 13-28). New York: Garland Science.

Dehaene, S. (2011). What Is Number? In The Number Sense: How the Mind Creates Mathematics (2nd ed., pp. 214-233). New York: Oxford University Press.

Euclid (1956a). The Thirteen Books of the Elements, Book 1 (2nd ed., Vol. 1, pp. 153-369, Heath, T.L., Trans.). New York: Dover Publications, Inc.

Euclid (1956b). The Thirteen Books of the Elements, Book 7(2nd ed., Vol. 2, pp. 277-344, Heath, T.L., Trans.). New York: Dover Publications, Inc.

El-Shamayleh, Y., \& Pasupathy, A. (2016). Contour Curvature as an Invariant Code for Objects in Visual Area V4. Journal of Neuroscience, 36, 5532-5543. 
https://doi.org/10.1523/JNEUROSCI.4139-15.2016

Heath, T. L. (2013). Theory of Proportion. In A History of Greek Mathematics (Vol. 1, pp. 325-327). Cambridge: Cambridge University Press.

Hilbert, D. (1950) The Foundations of Geometry. Townsend, E.J., Trans., La Salle, IL: Open Court Publishing Company

Hubel, D. H., \& Wiesel, T. N. (1959). Receptive Fields of Single Neurones in the Cat's Striate Cortex. Journal of Physiology, 148, 574-591. https://doi.org/10.1113/jphysiol.1959.sp006308

Hubel, D. H., \& Wiesel, T. N. (1968). Receptive Fields and Functional Architecture of Monkey Striate Cortex. Journal of Physiology, 195, 215-243. https://doi.org/10.1113/jphysiol.1968.sp008455

Kotani, K. (2016). Differential Calculus Based on Double Contradiction. Open Journal of Philosophy, 6, 420-427. https://doi.org/10.4236/ojpp.2016.64039

Kotani, K. (2017). What Is Number? Open Journal of Philosophy, 7, 116-125. https://doi.org/10.4236/ojpp.2017.72008

Nichollis, J. G., Martin, A. R., Wallace, B. G., \& Fuchs, P. A. (2002a). Universality of Electrical Signals. In From Neuron to Brain (4th ed., p. 10). Sunderland, MA: Sinauer Associates, Inc.

Nichollis, J. G., Martin, A. R., Wallace, B. G., \& Fuchs, P. A. (2002b). Signaling in the Lateral Geniculate Nucleus and Visual Cortex. In From Neuron to Brain (4th ed., pp. 407-425). Sunderland, MA: Sinauer Associates, Inc.

Pasupathy, A. (2015). The Neural Basis of Image Segmentation in the Primate Brain. Journal of Neuroscience, 296, 101-109.

https://doi.org/10.1016/j.neuroscience.2014.09.051

Plato (2007). Book VII. In The Republic (pp. 177-202). Jowett, B., Trans., New York: Dover Publications, Inc.

Tessier-Lavigne, M. (2000) Visual Processing by the Retina. In E. R. Kandel, J. H. Schwartz, \& T. M. Jessel (Eds.), Principles of Neural Science (4th ed., pp. 507-522). New York: McGraw-Hill, Health Professions Division.

Yue, X., Pourladian, I. S., Tootell, R. B. H., \& Ungerleider, L. G. (2014). Curvature-Processing Network in Macaque Visual Cortex. Proceedings of the National Academy of Sciences of the United States of America, 111, e3467-e3475.

https://doi.org/10.1073/pnas.1412616111

Wurtz, R. H., \& Kandel, E. R. (2000). Perception of Motion, Depth, and Form. In E. R. Kandel, J. H. Schwartz, \& T. M. Jessel (Eds.), Principles of Neural Science (4th ed., pp. 548-571). New York: McGraw-Hill, Health Professions Division. 